\section{Research Square \\ Preprints are preliminary reports that have not undergone peer review. \\ They should not be considered conclusive, used to inform clinical practice, or referenced by the media as validated information.}

\title{
Association between MTHFR C677T polymorphism and the susceptibility to nonsyndromic cleft lip with or without cleft palate in Chinese Han population: a case-control study and meta-analysis
}

Xuemei Lu

School of Medicine, Xiamen University

Xiaoling Deng

School of Medicine, Xiamen University

Tingying Liu

Department of Stomatology of Second Affiliated Hospital of Shantou University Medical College

\section{Qiufang Zhang}

Engineering Research Center of Fujian University for Stomatological Biomaterials, Department of Stomatology and Affiliated Stomatological Hospital of Xiamen Medical College

Mingyan Xu ( $\nabla$ myxu@xmu.edu.cn)

Engineering Research Center of Fujian University for Stomatological Biomaterials, Department of Stomatology and Affiliated Stomatological Hospital of Xiamen Medical College

\section{Research Article}

Keywords: MTHFR, Polymorphism, NSCL/P, Meta-analysis

Posted Date: May 12th, 2021

DOI: https://doi.org/10.21203/rs.3.rs-470556/v1

License: (c) (i) This work is licensed under a Creative Commons Attribution 4.0 International License. Read Full License 


\section{Abstract}

Background: Functional polymorphisms on methylenetetrahydrofolate reductase (MTHFR) gene are reported to be involved in the etiology of nonsyndromic cleft lip with or without cleft palate (NSCL/P), but the conclusions are still inconsistent. The aim of our study is to investigate the association between the susceptibility of nonsyndromic cleft lip with or without cleft palate (NSCL/P) and MTHFR C677T polymorphism in Chinese Han population $\bigotimes$

Methods: A case-control study was performed, followed by a Meta-analysis. Electronic databases including PubMed, Embase, CNKI, VIP and Wanfang database were searched for relevant literature from inception of databases to November 2020. Meta-analysis was performed using Revman 5.3 and STATA 12.0 software.

Results: The case-control study included 358 NSCL/P cases and 354 controls, which indicated that the variant T allele significantly increased NSCL/P risk in Southern China. Then, in the present Meta-analysis, a total of nine case-control studies with 1444 cases and 1555 controls were included. Overall, there was a significant association between MTHFR C677T polymorphism and NSCL/P susceptibility under all genetic models. The subgroup analysis of NSCL/P types showed significantly increased risk of CLO but not CPO or CLP. In terms of the stratified analysis by geographical location, a significantly association was observed in Southern China under all genetic models, while there was no significant association in Northern China. Sensitivity analyses, Begg's funnel plot and Egger's regression test further suggest the stable and trustworthy of these results.

Conclusion: MTHFR C677T polymorphism is associated with the risk of NSCL/P in Chinese Han population, especially increasing the risk of NSCL/P in southern Chinese Han populations.

\section{Background}

Nonsyndromic cleft lip with or without cleft palate (NSCL/P) can be broadly divided into cleft lip only (CLO), cleft palate only (CPO), and cleft lip and palate (CLP) based on clinical manifestation(Jugessur et al., 2009). NSCL/P is one of the most common congenital malformations in the world, and the incidence of NSCL/P varies greatly among different nationalities and geographical regions (Mossey and Modell 2012). In China, the live-birth prevalence was reported to reach 1.42/1000 (Dai et al., 2010).

NSCL/P is a genetic disease influenced by the interaction of genetic and environmental factors, and its underlying genetic causes and basic molecular mechanisms are still unclear (Dixon et al., 2011). Studies reported that perinatal intake of folic acid provides protection from NSCL/P.

Methylenetetrahydrofolate reductase (MTHFR) is an enzyme involved in folic acid metabolism, and MTHFR is also proved to be one of the several candidate genes (Alkuraya et al., 2006; Gaspar et al., 2004; Jagomägi et al., 2010; Moreno et al., 2009; Sull et al., 2009; Tamasas and Cox 2017; Zhu et al., 2010) that have been identified as the potential etiology of NSCL/P. The MTHFR gene is located at chromosome 1p36.3, contains 11 exons and spans approximately $2.2 \mathrm{~kb}$. MTHFR enzyme catalyzes the conversion of 5,10-methylenetetrahydrofolate to 5-methytetrahydrofolate in the process of folic acid metabolism in vivo. Several polymorphisms related to NSCL/P were found on MTHFR gene, among which the most common one is rs1801133, namely C677T. The MTHFR 677C>T polymorphism is a C to T transition at base pair 677(Frosst et al., 1995), and the encoded Alanine is replaced by Valine, which leads to the decrease of enzyme activity and thermal stability, affects the normal metabolism of intracellular folate, interferes with DNA synthesis and methylation, destroys the stability and integrity of the genome, and then causes a variety of birth defects (Weisberg et al., 1998).

Many researchers have conducted a large number of studies on MTHFR C677T polymorphism and NSCL/P susceptibility based on Chinese Han population, but the conclusions are inconsistent and there are many controversies. Therefore, a case-control study and meta-analysis on all eligible case-control studies were conducted in this study to summarize the results of previous studies, so as to systematically evaluate the correlation between MTHFR gene C677T polymorphism and NSCL/P susceptibility in Chinese Han population.

\section{Methods}

\subsection{Case-control study}

\subsection{Study population}

There are 358 NSCL/P cases and 354 controls in this case-control study. All cases together with controls were Han Chinese from Guangdong province recruited in the Second Affiliated Hospital of Shantou University between 2008 and 2011. The study was approved by the local ethics committee and the subjects' informed consent was obtained. The genetic analysis of the MTHFR C677T (rs1801133) polymorphism was performed by the matrix assisted laser desorption ionization time of flight mass spectrometry, MALDI-TOF-MS. Statistical analyses were conducted using SPSS 13.0 software as described (Niktabar et al., 2019): Hardy-Weinberg equilibrium (HWE) value of the control group was calculated by a goodness-of-fit $\chi^{2}$ test; differences in genotypes and alleles frequencies between NSCL/P cases and controls were compared by chi-squared ( $\left.\chi^{2}\right)$ test; the relative risk was assessed by Odds ratios (OR) and 95\% confidence interval (Cl); in addition, transmission disequilibrium test (TDT) analysis in Affected Person-Parent Trios was also performed. 


\subsection{Meta-analysis}

\subsection{Search strategy}

A systematic literature search was performed through the Electronic databases, which included PubMed, Embase, China National Knowledge Infrastructure (CNKI), VIP and Wanfang database. The last search was updated on November 22, 2020. The following keywords and terms were used: "nonsyndromic cleft lip with or without cleft palate", "NSCL/P", "MTHFR", "C677T" and "oral facial cleft". In addition, the reference lists of previous meta-analysis and relevant studies were also further searched to avoid missing some potential publications.

\subsection{Selection criteria}

All studies included in the meta-analysis met all the following inclusion criteria:(1) case-control study; (2) focused on the association between NSCL/P risk and the MTHFR C677T polymorphism in Chinese Han populations; and (3) provided sufficient data of genotype distributions in cases and controls. The following were exclusion criteria: (1) studies did not focus on humans or MTHFR C677T; (2) the genotype distributions in the control group deviated from those predicted by HWE; and (3) duplicate or overlapping study. If the study population is the same or overlapped and there is more than one paper, the recently published paper or the one with a large sample size was selected in the meta-analysis.

\subsection{Data extraction}

Original data were extracted from each eligible study by two authors independently according to the inclusion and exclusion criteria. The following data were extracted: the first author name; publication year; types of NSCL/P (CLO, CPO and CLP); geographical location; source of control; genotype distributions in cases and controls; genotype methods. When data were not available, authors were contacted by e-mail and asked to provide the missing information. The quality of the included studies was assessed according to the Newcastle-Ottawa Quality Assessment Scales (Stang 2010).

\subsection{Statistical analysis}

The strength of the association between MTHFR C677T polymorphism and NSCL/P risk was estimated using pooled OR with 95\% Cls. The statistical significance of pooled ORs was assessed by the $Z$ test, in which P-value $<0.05$ was considered as statistically significant. The strength of association was assessed under the following five genetic models: (1) allelic model (T vs. C); (2) homozygote model (TT vs. CC); (3) heterozygote model (CT vs. CC); (4) dominant model (CT + TT vs. CC); and (5) recessive model (TT vs. CC + CT). Heterogeneity across studies were measured by $\mathrm{I}^{2}$ statistics and $Q$ tests. When $\mathrm{P}>0.05$ or $\mathrm{I}^{2}<50 \%$, no significant heterogeneity was detected, and a fixed-effect model was implemented, otherwise, the random-effect model was performed. In addition, subgroup analyses were conducted according to the NSCL/P type (CLO, CPO and CLP) and geographical location (Northern China and Southern China). The sensitivity analysis was conducted to evaluate the stability of the results through omission of each study in turn. The Begg's test and Egger's test were used to assess the publication bias, and $\mathrm{P}<0.05$ was considered to indicate statistically significant publication bias. All statistical tests were performed using Revman 5.3 software (Nordic Cochrane Center, Copenhagen, Denmark) and STATA 12.0 software (STATA Corp, College Station, TX, USA).

\section{Results}

\subsection{Case-control study}

The genotype frequencies of MTHFR C677T polymorphism were in agreement with the HWE (Table 1). Table 2 displays the distribution of MTHFR C677T polymorphism and estimated ORs in NSCL/P cases and control subjects. In CLO cases, there were statistically significant differences of genotypes and allele distribution in comparing with controls, while no significant difference was observed between CPO or CLP cases and controls $(P>0.05)$. The results of genetic model analysis suggested that MTHFR C677T polymorphism was associated with the risk of CLO in the homozygous model, while no association with NSCL/P susceptibility was found in other gene models. In addition, the nuclear families with heterozygous information were analyzed by TDT test. As shown in Table 3, at the rs1801133 site, there was transmission imbalance, and the T allele was overtransmitted in the patients with cleft lip and palate $(\mathrm{P}<0.001)$.

Table 1 Tests of Hardy-Weinberg equilibrium in MTHFR C677T among cases and Control

\begin{tabular}{cccccc}
\hline & NSCL/P & CLO & CPO & CLP & Control \\
\hline HWE $\chi^{2}$ & 2.713 & 2.580 & 1.452 & 2.516 & 0.031 \\
P value & 0.100 & 0.108 & 0.228 & 0.113 & 0.860 \\
\hline
\end{tabular}

Table 2 Genotypic and Allelic Distributions of MTHFR C677T polymorphism in Cases and Control Individuals 


\begin{tabular}{|c|c|c|c|c|c|}
\hline & \multicolumn{4}{|c|}{ Members of families with Cleft } & \multirow{3}{*}{$\begin{array}{l}\text { Controls } \\
\mathrm{N}=354\end{array}$} \\
\hline & NSCL/P & CLO & $\mathrm{CPO}$ & CLP & \\
\hline & $\mathrm{N}=358$ & $\mathrm{~N}=69$ & $\mathrm{~N}=59$ & $\mathrm{~N}=230$ & \\
\hline \multicolumn{6}{|l|}{ Genotypes } \\
\hline $\mathrm{CC}$ & $200(55.9 \%)$ & $33(47.8 \%)$ & $28(47.5 \%)$ & $139(60.4 \%)$ & $197(55.7 \%)$ \\
\hline CT & $127(35.5 \%)$ & $25(36.2 \%)$ & $28(47.5 \%)$ & $74(32.2 \%)$ & $135(38.1 \%)$ \\
\hline TT & $31(8.6 \%)$ & $11(16 \%)$ & $3(5 \%)$ & $17(7.4 \%)$ & $22(6.2 \%)$ \\
\hline$\chi^{2}(\mathrm{P}, \mathrm{df}=2)$ & $1.773(0.412)$ & $7.709(0.021)$ & $1.843(0.398)$ & $2.229(0.328)$ & \\
\hline \multicolumn{6}{|c|}{ Odds ratio $(95 \% \mathrm{CI})$ for comparison with controls } \\
\hline TT vs CC & $1.39[0.78,2.48]$ & $2.98[1.32,6.72]$ & $0.96[0.27,3.41]$ & $1.10[0.56,2.14]$ & \\
\hline CT vs CC & $0.93[0.68,1.27]$ & $1.11[0.63,1.94]$ & $1.46[0.83,2.57]$ & $0.78[0.54,1.11]$ & \\
\hline $\mathrm{CT}+\mathrm{TT}$ vs CC & $0.99[0.74,1.33]$ & $1.37[0.82,2.30]$ & $1.39[0.80,2.41]$ & $0.82[0.59,1.15]$ & \\
\hline \multicolumn{6}{|l|}{ Alleles } \\
\hline $\mathrm{C}$ & $527(73.6 \%)$ & $91(65.9 \%)$ & $84(71.2 \%)$ & $352(76.5 \%)$ & $529(74.7 \%)$ \\
\hline $\mathrm{T}$ & $189(26.4 \%)$ & $47(34.1 \%)$ & $34(28.8 \%)$ & 108(23.5\%) & $179(25.3 \%)$ \\
\hline$\chi^{2}(\mathrm{P}, \mathrm{df}=1)$ & $0.231(0.631)$ & $4.543(0.033)$ & $0.659(0.417)$ & $0.490(0.484)$ & \\
\hline
\end{tabular}

Bold values are significant results.

Table 3 TDT Analysis of MTHFR C677T in Affected Person-Parent Trios

\begin{tabular}{ccccc}
\hline Alleles & Transmitted & Not transmitted & $\chi^{2}$ & P value \\
\hline $\mathrm{C}$ & 84 & 30 & 25.579 & $<0.001$ \\
$\mathrm{~T}$ & 30 & 84 & & \\
\hline
\end{tabular}

\subsection{Meta-analysis}

\subsection{Characteristics of selected studies}

Fig. 1 shows flowchart of the study selection in this meta-analysis. All studies were screened based on the preset inclusion and exclusion criteria. Final nine case-control studies with 1444 NSCL/P cases and 1555 controls were selected in the meta-analysis. The main characteristics including the first author name, publication year, types of NSCL/P (CLO, CPO and CLP), geographical location, source of control, genotype distributions in cases and controls, genotype methods, the HWE value of the control group as well as the NOS evaluation results of the each study are summarized in Table 4 . Moreover, in all case-control studies, the distributions of genotype of the controls were in agreement with $H W E(P>0.05)$.

Table 4 Characteristics of studies included in the meta-analysis

\begin{tabular}{|c|c|c|c|c|c|c|c|c|}
\hline \multirow[t]{2}{*}{ Study (year) } & \multirow{2}{*}{$\begin{array}{l}\text { Geographical } \\
\text { location }\end{array}$} & \multirow{2}{*}{$\begin{array}{l}\text { Source of } \\
\text { control }\end{array}$} & \multirow{2}{*}{$\begin{array}{l}\text { Type of } \\
\text { disease }\end{array}$} & \multicolumn{2}{|c|}{$\mathrm{a}_{\text {Genotype }}$} & \multirow{2}{*}{$\begin{array}{l}\text { Genotype } \\
\text { method }\end{array}$} & \multirow{2}{*}{$\begin{array}{l}\text { P for } \\
\text { HWE }\end{array}$} & \multirow[t]{2}{*}{ NOS } \\
\hline & & & & Case & trol & & & \\
\hline Deng et al. (2014) & South China & HB & NSCL/P & $32 / 73 / 19$ & $148 / 104 / 21$ & PCR-RFLP & 0.648 & 6 \\
\hline Du et al.(2016) & North China & PB & NSCL/P & $16 / 51 / 29$ & $36 / 40 / 24$ & PCR-RFLP & 0.060 & 5 \\
\hline Guo et al. (2009) & North China & HB & NSCL/P & $19 / 53 / 24$ & $22 / 57 / 24$ & PCR & 0.277 & 5 \\
\hline \multirow[t]{4}{*}{ Han et al. (2011) } & South China & HB & NSCL/P & $50 / 111 / 36$ & $74 / 110 / 29$ & PCR-RFLP & 0.236 & 5 \\
\hline & & & CPO & $4 / 5 / 1$ & - & & & \\
\hline & & & CLO & $20 / 54 / 16$ & - & & & \\
\hline & & & CLP & $26 / 52 / 19$ & - & & & \\
\hline \multirow[t]{4}{*}{ Ren et al. (2012) } & North China & HB & NSCL/P & $243 / 84 / 7$ & $207 / 98 / 9$ & PCR-RFLP & 0.521 & 6 \\
\hline & & & $\mathrm{CPO}$ & $85 / 22 / 3$ & - & & & \\
\hline & & & CLO & $75 / 32 / 1$ & - & & & \\
\hline & & & CLP & $83 / 30 / 3$ & - & & & \\
\hline Wan et al. (2006) & South China & $\mathrm{HB}$ & NSCL/P & $13 / 49 / 14$ & $31 / 20 / 9$ & PCR-RFLP & 0.075 & 6 \\
\hline \multirow[t]{4}{*}{ Wang et al. (2012) } & South China & HB & NSCL/P & $9 / 50 / 15$ & $24 / 35 / 15$ & PCR-RFLP & 0.732 & 6 \\
\hline & & & $\mathrm{CPO}$ & $1 / 4 / 0$ & - & & & \\
\hline & & & CLO & $3 / 18 / 10$ & - & & & \\
\hline & & & CLP & $5 / 28 / 5$ & - & & & \\
\hline $\begin{array}{l}\text { Wang SM et al. } \\
(2012)\end{array}$ & North China & HB & NSCL/P & $10 / 41 / 38$ & $15 / 39 / 10$ & PCR-RFLP & 0.070 & 5 \\
\hline \multirow[t]{4}{*}{ Xu et al.(2020) } & South China & HB & NSCL/P & $200 / 127 / 31$ & $197 / 135 / 22$ & MALDI-TOF-MS & 0.860 & 6 \\
\hline & & & CPO & $28 / 28 / 3$ & - & & & \\
\hline & & & CLO & $33 / 25 / 11$ & - & & & \\
\hline & & & CLP & $139 / 74 / 17$ & - & & & \\
\hline
\end{tabular}

HB, hospital based; PB, population based.

${ }^{\mathrm{a}}$ Genotype, for rs1801133, CC/CT/TT; HWE, Hardy-Weinberg equilibrium; PCR: polymerase chain reaction;

RFLP: restriction fragment length polymorphism.

\subsection{Association between the MTHFR C677T polymorphism and NSCL/P risk}

The summary of the meta-analysis of the association of MTHFR C677T polymorphism with NSCL/P risk is shown in Table 6.The pooled results revealed that the MTHFR C677T polymorphism was significantly associated with an increased risk of NSCL/P in Chinese Han populations under all the genetic models: allelic model ( $\mathrm{T}$ vs. $\mathrm{C}, \mathrm{OR}=1.43,95 \% \mathrm{Cl}=1.11-1.86, \mathrm{P}=0.006$, Fig. 2A), homozygote model $(T T$ vs. $\mathrm{CC}, \mathrm{OR}=2.14,95 \% \mathrm{Cl}=1.43-$ 
3.22, $\mathrm{P}=0.0002$, Fig. 2B), heterozygote model ( $\mathrm{CT}$ vs. $\mathrm{CC}, \mathrm{OR}=1.82,95 \% \mathrm{Cl}=1.14-2.89, \mathrm{P}=0.01$, Fig. $2 \mathrm{C}$ ), dominant model $(\mathrm{CT}+\mathrm{TT}$ vs. $\mathrm{CC}, \mathrm{OR}=1.90$, $95 \% \mathrm{Cl}=1.20-2.99, \mathrm{P}=0.006$, Fig. $2 \mathrm{D}$ ) and recessive model (TT vs. $\mathrm{CC}+\mathrm{CT}, \mathrm{OR}=1.48,95 \% \mathrm{Cl}=1.18-1.85, \mathrm{P}=0.0007, \mathrm{Fig} .2 \mathrm{E}$ ).

\subsection{Subgroup analysis of NSCL/P types}

A stratified analysis was performed by NSCL/P types (CLO, CPO and CLP). According to the results, in CLO patients, a significant association between MTHFR C677T polymorphism and CLO risk was observed under homozygote model (TT vs. CC, OR =2.18, 95\% $\mathrm{Cl}=1.34-3.55, \mathrm{P}=0.002$ ) and recessive model (TT vs. $C C+C T, O R=1.61,95 \% \mathrm{Cl}=1.05-2.46, \mathrm{P}=0.03)$. For $\mathrm{CPO}$ and CLP patients, there was no association between the MTHFR C677T polymorphism and CPO or CLP risk (Table 5).

\subsection{Subgroup analysis of geographical location}

We also conducted a subgroup analysis by geographical location (Southern China and Northern China). In general, five studies (Wang et al., 2012a; Wan et al., 2006; Han et al., 2011; Deng et al., 2014), including current study, belong to Southern China, and four studies (Du et al., 2016; Wang et al., 2012b; Ren and Nan 2012; Guo et al., 2009) belong to Northern China. The results indicated that MTHFR C677T polymorphism was associated with a significantly increased NSCL/P susceptibility in the Southern Chinese Han populations under all five genetic models, while no correlation in Northern Chinese Han populations was found in any of the genetic models (Table 5).

\subsection{Sensitivity analysis}

A sensitivity analysis was conducted to assess the stability of the meta-analysis by orderly removing individual studies. No single study greatly affected the overall pooled ORs (data not shown), indicating the stability and reliability of our results.

\subsection{Publication bias}

Begg's funnel plot and Egger's linear regression test were conducted to assess the potential publication bias. Fig. 3A-E and Fig. 4A-E show the results under the five genetic models. There was no publication bias between the NSCL/P risk and the polymorphism in the genetic models: $P$ values of both Begg's and Egger's tests were more than 0.05 , except for the Egger's test of heterozygote model $(P=0.033)$ and dominant model $(P=0.028)$ revealed a publication bias (Table 6).

Table 5 NSCL/P and subgroup result of the association with MTHFR C677T polymorphism.

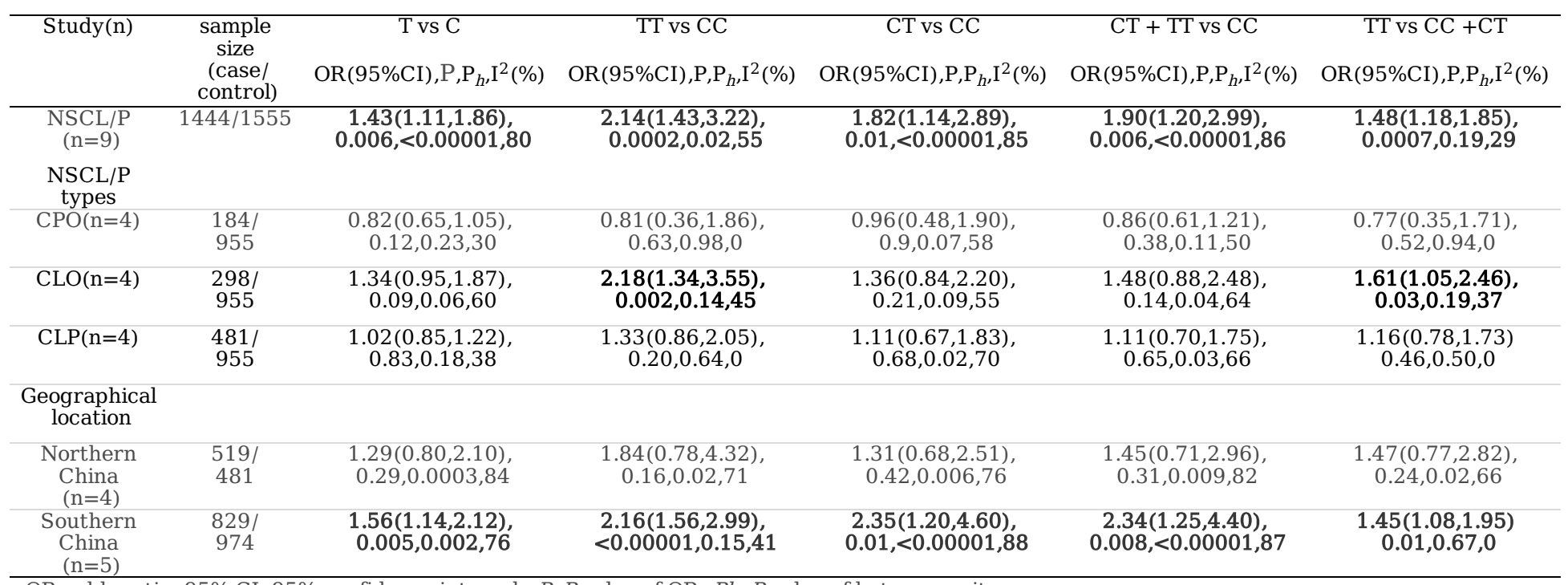

OR, odds ratio; 95\% CI, 95\% confidence interval; $P, P$-value of OR; $P h, P$-value of heterogeneity.

Bold values are significant results in the meta-analysis.

Table 6 The publication bias results of the association with MTHFR C677T polymorphism.

\begin{tabular}{lllll}
\hline & \multicolumn{2}{c}{ Begg's test } & \multicolumn{2}{c}{ Egger's test } \\
\cline { 2 - 5 } Genetic model & $\mathrm{Z}$ & $\mathrm{P}$ & $\mathrm{t}$ & $\mathrm{P}$ \\
\hline T vs C & 1.56 & 0.118 & 1.71 & 0.130 \\
TT vs CC & 0.94 & 0.348 & 0.74 & 0.484 \\
\hline CT vs CC & 1.15 & 0.251 & 2.65 & 0.003 \\
\hline CT + TT vs CC & 1.15 & 0.251 & 2.76 & 0.028 \\
\hline TT vs CC +CT & 1.15 & 0.251 & -0.31 & 0.766 \\
\hline
\end{tabular}

\section{Discussion}


The pathogenesis of NSCL/P has not been fully elucidated. It is generally believed that NSCL/P is a complex malformation and genetically susceptible disease affected by both gene and environment, and gene polymorphism variation is one of the main etiologies. Identifying the potential risk factors can contribute to identify high-risk populations and promote the development of preventive therapies for NSCL/P.

A number of genes are reported to be involved in the etiology of NSCL/P, such as IRF6, MSX1, MTHFR, FOXE1 and TGF-beta. Among those genes, MTHFR is the most validated gene. However, publications regarding the association between MTHFR and NSCL/P were inconsistent. Some studies (Li et al., 2020; Luo et al., 2012; Pan et al., 2015; Pan et al., 2012; Rai 2018; Shu et al., 2019; Zhao et al., 2014) reported that MTHFR C677T polymorphism was significantly associated with NSCL/P risk in Asian, Caucasian and Mixed populations, whereas Lmani et al.(Imani et al., 2020) found that there was no significant association based on the ethnicity or the source of cases. And according to Amooee's research (Amooee et al., 2019), the result of stratified analysis by country of origin revealed a significant association in Brazilian, Turkish and Indian populations, but not in US-American and Chinese.

Several studies have investigated the association between MTHFR C677T polymorphism and NSCL/P risk specifically in Chinese Han populations. But the results were also inconsistent due to the differences on population, study design, family history, and statistical analysis. In this case-control study, we have evaluated the association between MTHFR C677T polymorphism and NSCL/P risk in Chinese Southern population. We found there was a significant difference of genotypes and allele distribution between CLO cases and controls $(\mathrm{P}<0.05)$, but not between CPO or CLP cases and controls $(P>0.05)$. In addition, the TDT analysis indicated the T allele has been overtransmitted in patients with NSCL/P $(P<0$. 001). The results of our casecontrol study are consistent with the previous studies (Wang et al., 2012a; Han et al., 2011; Deng et al., 2014).

Based on the results, we next conducted an updated meta-analysis to further evaluate the strength of the association. Nine case-control studies with a total of 1444 cases and 1555 controls were finally included. We observed that MTHFR C677T polymorphism was associated with NSCL/P susceptibility in Chinese Han population under all genetic models. Moreover, stratified analysis by NSCL/P types showed that MTHFR C677T polymorphism was significantly associated with an increased risk of CLO, but not CPO or CLP. Subgroup analysis by geographical location indicated that the MTHFR C677T polymorphism played different roles in the south and north of China. In Southern China, individuals carrying the mutant allele might have higher risk of NSCL/P, whereas in Northern China, no significant association was found.

Although several meta-analysis studies have been published to quantitatively evaluate the correlation between MTHFR gene C677T polymorphism and NSCL/P risk in Chinese Han population(Zhu et al., 2013; Wang et al., 2016; Qin et al., 2013; Ma et al., 2018; Jiang et al., 2014), the conclusions were still controversial. Moreover, subgroup analysis based on different phenotypes and geographic locations of NSCL/P has not been reported. Our metaanalysis evaluated the correlation between the MTHFR C677T polymorphism and NSCL/P susceptibility in Chinese Han populations comprehensively. In addition, subgroup analysis was conducted based on different NSCL/P phenotypes and geographical locations to investigate the potential differences of correlation between CPO, CLO, CLP and populations in the south and north of China. Sensitivity analysis, Begg's funnel plot and Egger's regression test further suggest the stable and trustworthy of our results.

Some limitations still should be considered in this meta-analysis. First, the sources of control and genotype methods were vary from one to another. Second, NSCL/P is a multifactorial and complex malformation resulted from genetic and environmental interactions. However, we only explored the association between the MTHFR C677T polymorphism and NSCL/P risk in Chinese Han populations in this study without further investigation of gene environment or gene-gene interactions. Third, there were differences in baseline data, such as inconsistencies in case and control selection criteria and mismatch in age or gender composition of study subjects. Finally, some large significant heterogeneity might exist in most genetic models, although we performed subgroup analyses according to NSCL/P types and geographical location. The factors above affect the reliability of meta-analysis results to some extent.

In conclusion, the present meta-analysis revealed that MTHFR C677T polymorphism was associated with an increased risk of NSCL/P in Chinese Han populations. However, it is still necessary to optimize the design, expand the sample size for further research, and take into account the potential interaction between genes and between genes and environment, so as to obtain more accurate and reliable conclusions.

\section{List Of Abbreviations}

NSCL/P: Non-syndromic cleft lip with or without cleft palate; CLO: cleft lip only; CPO: cleft palate only; CLP: cleft lip and palate; MTHFR:

Methylenetetrahydrofolate reductase; MALDI-TOF-MS: the matrix assisted laser desorption ionization time of flight mass spectrometry; $\chi 2$ : chi-squared; OR: Odds ratio; Cl: Confidence interval; TDT: transmission disequilibrium test; CNKI: The China National Knowledge Internet; WF: Wanfang; NOS: NewcastleOttawa scale; T vs. C: allelic model; TT vs. CC: homozygote model; CT vs. CC: heterozygote model; CT + TT vs. CC: dominant model; TT vs. CC + CT: recessive model.

\section{Declarations}

\section{Ethics approval and consent to participate}

This case-control study was approved by the local ethics committee and the subjects' informed consent was obtained. This meta-analysis is conducted by the data from previously published papers, ethical approval and patient consent are not required. 


\section{Consent for publication}

Not applicable.

\section{Availability of data and materials}

The datasets generated and analyzed during thte current study are available from the corresponding author on reasonable request.

\section{Competing interests}

The authors declare that they have no competing interests.

\section{Funding}

This work was supported by National Natural Science Foundation of China (NO. 81671001 and NO. 81072208 to Mingyan Xu, NO. 82071128 to Xiaoling Deng).

\section{Authors' contributions}

Xuemei Lu contributed to conceptualization, methodology, data collecting, result analysis, and writing original article; Tingying Liu and Qiufang Zhang contributed to methodology and literature search; Xiaoling Deng and Mingyan Xu contributed to review writing and editing, supervision, and funding acquisition.

\section{Acknowledgements}

The authors thank all the study subjects for their participation and personnel in the hospitals for their assistance.

\section{References}

Jugessur, A., Farlie, P.G., Kilpatrick, N. (2009) The genetics of isolated orofacial clefts: from genotypes to subphenotypes. Oral diseases. 15:437-53

Mossey, P.A., Modell, B. (2012) Epidemiology of oral clefts 2012: an international perspective. Frontiers of oral biology. 16:1-18

Dai, L., Zhu, J., Mao, M., Li, Y., Deng, Y., Wang, Y., et al. (2010) Time trends in oral clefts in Chinese newborns: data from the Chinese National Birth Defects Monitoring Network. Birth Defects Res A Clin Mol Teratol. 88:41-7

Dixon, M.J., Marazita, M.L., Beaty, T.H., Murray, J.C. (2011) Cleft lip and palate: understanding genetic and environmental influences. Nature reviews Genetics. 12:167-78

Alkuraya, F.S., Saadi, I., Lund, J.J., Turbe-Doan, A., Morton, C.C., Maas, R.L. (2006) SUM01 haploinsufficiency leads to cleft lip and palate. Science (New York, NY). 313:1751

Gaspar, D.A., Matioli, S.R., de Cássia Pavanello, R., Araújo, B.C., Alonso, N., Wyszynski, D., et al. (2004) Maternal MTHFR interacts with the offspring's BCL3 genotypes, but not with TGFA, in increasing risk to nonsyndromic cleft lip with or without cleft palate. European journal of human genetics :

EJHG. 12:521-6

Jagomägi, T., Nikopensius, T., Krjutskov, K., Tammekivi, V., Viltrop, T., Saag, M., et al. (2010) MTHFR and MSX1 contribute to the risk of nonsyndromic cleft lip/palate. European journal of oral sciences. 118:213-20

Moreno, L.M., Mansilla, M.A., Bullard, S.A., Cooper, M.E., Busch, T.D., Machida, J., et al. (2009) FOXE1 association with both isolated cleft lip with or without cleft palate, and isolated cleft palate. Hum Mol Genet. 18:4879-96

Sull, J.W., Liang, K.Y., Hetmanski, J.B., Wu, T., Fallin, M.D., Ingersoll, R.G., et al. (2009) Evidence that TGFA influences risk to cleft lip with/without cleft palate through unconventional genetic mechanisms. Human genetics. 126:385-94

Tamasas, B., Cox, T.C. (2017) Massively Increased Caries Susceptibility in an Irf6 Cleft Lip/Palate Model. Journal of dental research. 96:315-22

Zhu, J., Hao, L., Li, S., Bailey, L.B., Tian, Y., Li, Z. (2010) MTHFR, TGFB3, and TGFA polymorphism and their association with the risk of non-syndromic cleft lip and cleft palate in China. Am J Med Genet A. 152a:291-8

Frosst, P., Blom, H.J., Milos, R., Goyette, P., Sheppard, C.A., Matthews, R.G., et al. (1995) A candidate genetic risk factor for vascular disease: a common mutation in methylenetetrahydrofolate reductase. Nature genetics. 10:111-3

Weisberg, I., Tran, P., Christensen, B., Sibani, S., Rozen, R. (1998) A second genetic polymorphism in methylenetetrahydrofolate reductase (MTHFR) associated with decreased enzyme activity. Molecular genetics and metabolism. 64:169-72 
Niktabar, S.M., Aarafi, H., Dastgheib, S.A., Noorishadkam, M., Mirjalili, S.R., Lookzadeh, M.H., et al. (2019) Association of MTHFR 1298A > C Polymorphism with Susceptibility to Non-Syndromic Cleft Lip with or without Palate: A Case-Control Study and Meta-Analysis. Fetal and pediatric pathology.1-17

Stang, A. (2010) Critical evaluation of the Newcastle-Ottawa scale for the assessment of the quality of nonrandomized studies in meta-analyses. European journal of epidemiology. 25:603-5

Wang, X.Y., Li, G.Z., Tang, L.J. (2012a) The Related Study between MTHFR-677C/T Gene Polymorphism and NSCL/P in the Northern Part of Anhui Province. Chinese Journal of General Practice.

Wan, W.D., Wang, L.J., Zhou, X.P., Zhou, D.L., Zhang, Q.G., Huang, J.L., et al. (2006) Relationship between nonsyndromic cleft lip with or without cleft palate (NSCL/P) and genetic polymorphism of MTHFR C677T and A1298C. Chinese Journal of Plastic Surgery. 22:8-11

Han, Y., Pan, Y., Du, Y., Tong, N., Wang, M., Zhang, Z., et al. (2011) Methylenetetrahydrofolate reductase C677T and A1298C polymorphism and nonsyndromic orofacial clefts susceptibility in a southern Chinese population. DNA Cell Biol. 30:1063-8

Deng, Y.Q., Zhang, J., Zhu, Y.M., Zheng, C.S. (2014) Correlation between MTHFR Gene C677T Polymorphism and the Susceptibility of NSCL/P in Guangdong Population. Shenzhen Journal of Integrated Traditional Chinese and Western Medicine.

Du, P.G., Riang, B., Qu, W.D., Liu, H., Shang, J., Liu, Z.H. (2016) Research of dependablity between gene mutation of MTHFR C677T and CBS T833C and Non-syndromic cleft lip with or without cleft palate. General Journal of Stomatology. 3:12-4

Wang, S.M., Wang, J.H., Yu, J.C., Wei, B., Wang, K.H., Liu, J.Y., et al. (2012b) Association between parental MTHFR gene polymorphism 677C/T and nonsyndromic cleft lip and palate in offspring. Zhonghua yi xue yi chuan xue za zhi = Zhonghua yixue yichuanxue zazhi = Chinese journal of medical genetics. 29:464-7

Ren, Y., Nan, X.R. (2012) rs1801133 Polymorphism of MTHFR Gene Negatively Associated with Non-syndromic Cleft Lip with or without Cleft Palate in Shanxi Population. Journal of Oral and Maxillofacial Surgery.

Guo, J.Z., Song, X.M., Wang, Y., Zhu, W.L., Li, S.Q., Li, Y. (2009) Relationship between genetic polymorphism of MTHFR C677T and nonsyndromic cleft lip with or without palate. JOURNAL OF PEKINGUN IVERSITY(HEALTH SCIENCES). 41:432-6

Li, Q., Xu, L., Jia, X., Saleem, K., Zaib, T., Sun, W., et al. (2020) SNPs in folate pathway are associated with the risk of nonsyndromic cleft lip with or without cleft palate, a meta-analysis. Bioscience reports. $\mathbf{4 0}$

Luo, Y.L., Cheng, Y.L., Ye, P., Wang, W., Gao, X.H., Chen, Q. (2012) Association between MTHFR polymorphism and orofacial clefts risk: a meta-analysis. Birth Defects Res A Clin Mol Teratol. 94:237-44

Pan, X., Wang, P., Yin, X., Liu, X., Li, D., Li, X., et al. (2015) Association between Maternal MTHFR Polymorphism and Nonsyndromic Cleft Lip with or without Cleft Palate in Offspring, A Meta-Analysis Based on 15 Case-Control Studies. International journal of fertility \& sterility. 8:463-80

Pan, Y., Zhang, W., Ma, J., Du, Y., Li, D., Cai, Q., et al. (2012) Infants' MTHFR polymorphism and nonsyndromic orofacial clefts susceptibility: a metaanalysis based on 17 case-control studies. Am J Med Genet A. 158a:2162-9

Rai, V. (2018) Strong Association of C677T Polymorphism of Methylenetetrahydrofolate Reductase Gene With Nosyndromic Cleft Lip/Palate (nsCL/P). Indian journal of clinical biochemistry : IJCB. 33:5-15

Shu, X., Shu, S., Yang, L. (2019) Association between methylenetetrahydrofolate reductase polymorphism and non-syndromic cleft lip with or without palate susceptibility: an updated systematic review and meta-analysis. The British journal of oral \& maxillofacial surgery. 57:819-30

Zhao, M., Ren, Y., Shen, L., Zhang, Y., Zhou, B. (2014) Association between MTHFR C677T and A1298C polymorphism and NSCL/P risk in Asians: a meta-analysis. PLoS One. 9:e88242

Imani, M.M., Golchin, N., Safaei, M., Rezaei, F., Abbasi, H., Sadeghi, M., et al. (2020) Methylenetetrahydrofolate reductase C677T polymorphism is not associated with the risk of nonsyndromic cleft lip/palate: An updated meta-analysis. Sci Rep. 10:1531

Amooee, A., Dastgheib, S.A., Niktabar, S.M., Noorishadkam, M., Lookzadeh, M.H., Mirjalili, S.R., et al. (2019) Association of Fetal MTHFR 677C > T Polymorphism with Non-Syndromic Cleft Lip with or without Palate Risk: A Systematic Review and Meta-Analysis. Fetal and pediatric pathology.1-17

Zhu, B., Wang, D., Yang, B.Y., Liu, Y.Y., Li, Y.F., Sun, G.F. (2013) Relationship between 5,10-methylenetrahydrofolate Reductase C677T Polymorphism and Non-syndrome Cleft Lip and Palate in China: A Meta-analysis. Chinese General Practice. 16:3825-7

Wang, W., Luo, Y.L., Cheng, Y.L. (2016) Meta analysis of association between MTHF囚 gene polymorphism and nonsyndromic cleft lip and palate in Chinese Han population. Maternal and Child Health Care of China. 031:672-4

Page 8/12 
Qin, G., Wang, Q.X., Liu, W.X. (2013) Association between genetic polymorphism of MTHFR C677T and susceptibility of nonsymdromic cleft lip with or without palate: a Meta-analysis. Chinese Journal of Practical Pediatrics

Ma, H.H., Cha, J., Zhang, X., Shi, H.C., Wang, Y.J., Chen, Y. (2018) 5,10-MTHF囚 gene polymorphism and susceptibility to nonsyndromic cleft lip and palate in Asian population: A meta-analysis. J Pract Stomatol. 34:95-9

Jiang, C., Yin, N., Wu, Z.Z.D., Wang, Y., Li, H., Song, T. (2014) Lack of Association Between MTHFR, MTR, MTRR, and TCN2 Genes and Nonsyndromic $\mathrm{CL} \pm \mathrm{P}$ in a Chinese Population: Case-Control Study and Meta-Analysis. The Cleft palate-craniofacial journal

\section{Figures}

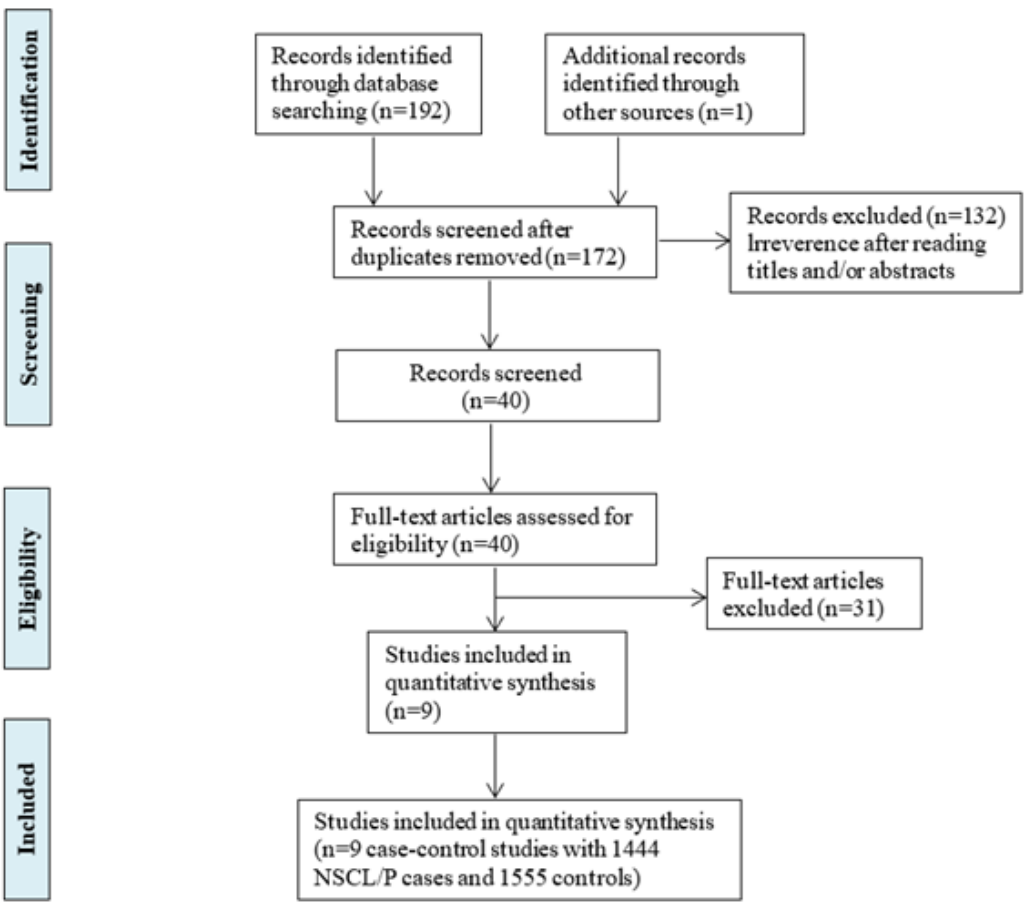

Figure 1

Flowchart of the study selection in this meta-analysis. 
A

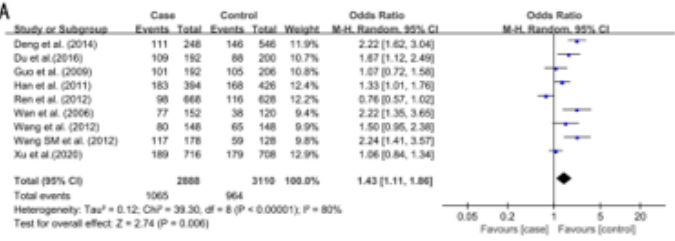

B

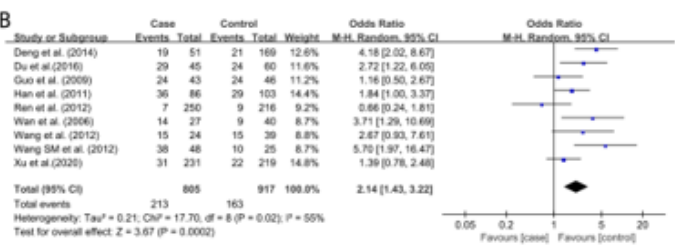

C

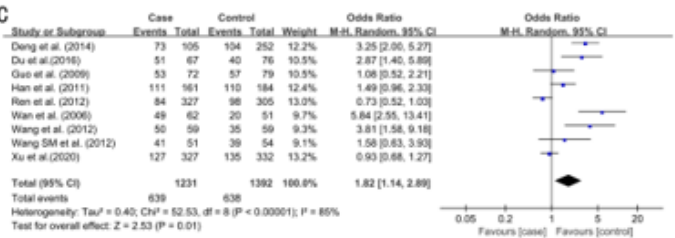

D

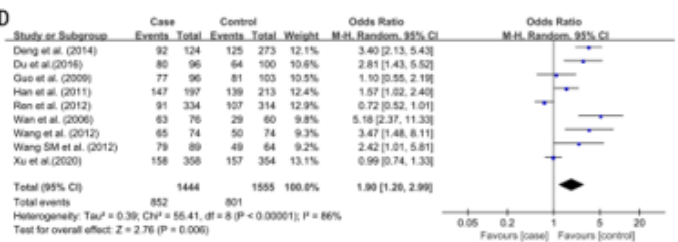

E

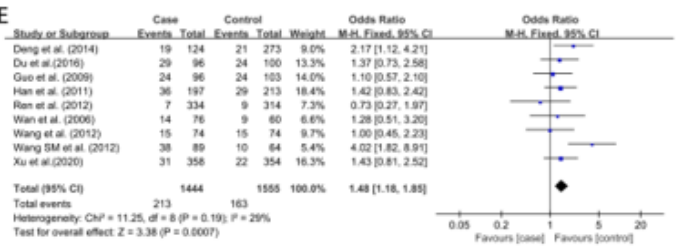

\section{Figure 2}

Forest plot of NSCL/P susceptibility associated with MTHFR C677T polymorphism in Chinese Han populations based on five genetic models: (A) allelic model (T vs. C),(B) homozygote model(TT vs. CC), (C) heterozygote model (CT vs. CC), (D) dominant model (CT + TT vs. CC), (E) recessive model (TT vs. $\mathrm{CC}+\mathrm{CT})$. 


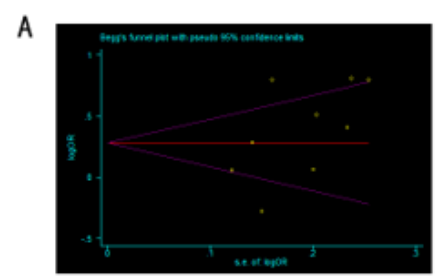

B

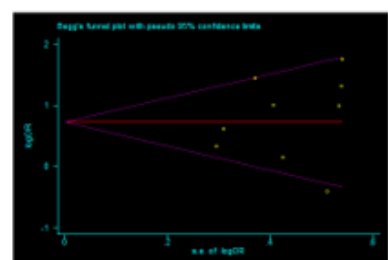

C

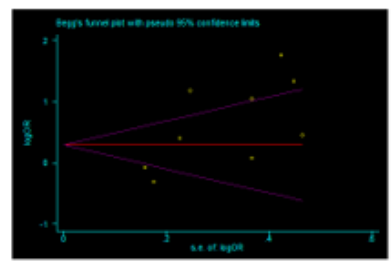

D

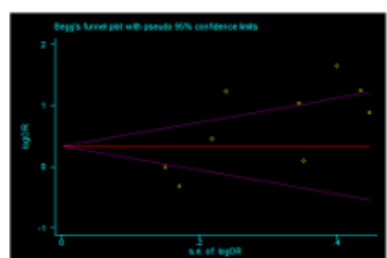

$\mathrm{E}$

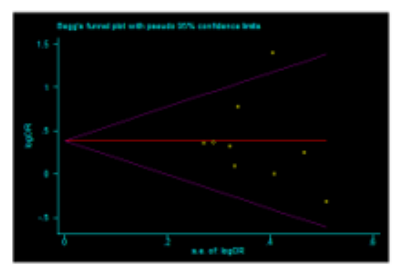

\section{Figure 3}

Begg's funnel plot to detect publication bias for MTHFR C677T polymorphism and the risk of NSCL/P in Chinese Han populations:(A) allelic model ( $T$ vs. C),(B) homozygote model(TT vs. CC), (C) heterozygote model (CT vs. CC), (D) dominant model (CT + TT vs. CC), (E) recessive model (TT vs. CC + $\mathrm{CT})$. 


\section{A}

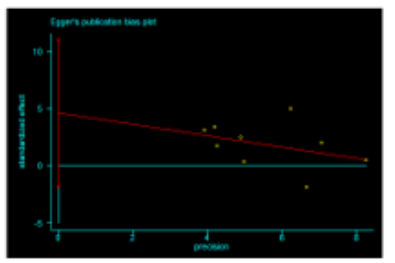

B

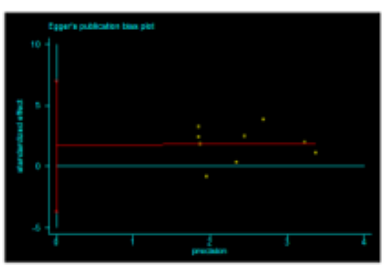

C

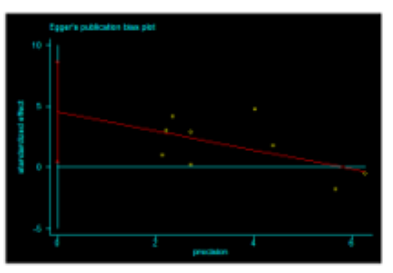

D

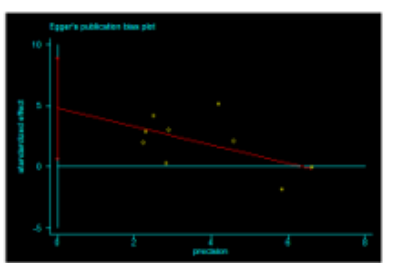

E

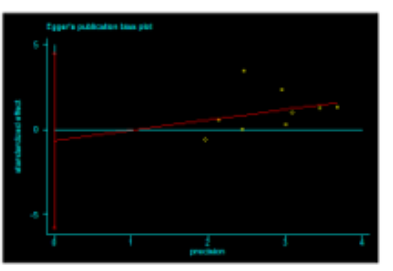

Figure 4

Egger's test to detect publication bias for MTHFR C677T polymorphism and the risk of NSCL/P in Chinese Han populations: (A) allelic model (T vs. C), (B) homozygote model(TT vs. CC), (C) heterozygote model (CT vs. CC), (D) dominant model (CT + TT vs. CC), (E) recessive model (TT vs. CC + CT). 